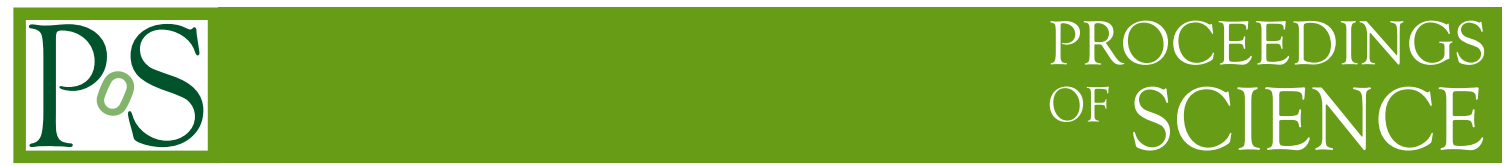

\title{
Projected Performance of CMS Cathode Strip Chambers at HL-LHC
}

\section{Bhargav Joshi}

On behalf of CMS Muon Group

University of Florida, Gainesville, FL, USA

E-mail: bm163@ufl. edu

Cathode Strip Chambers (CSCs) are a crucial component of the CMS endcap muon system which will operate throughout the lifetime of the LHC and beyond, during the HL-LHC running. We present an analysis of the expected CSC performance in a HL-LHC like environment as well as studies of CSC detector longevity over the lifetime of the HL-LHC.

Sixth Annual Conference on Large Hadron Collider Physics (LHCP2018)

4-9 June 2018

Bologna, Italy 


\section{Introduction}

High Luminosity LHC (HL-LHC) [1] is expected to deliver luminosity starting at $5 \times 10^{34}$ $\mathrm{cm}^{-2} \mathrm{~s}^{-1}$ and reaching a value as high as $7.5 \times 10^{34} \mathrm{~cm}^{-2} \mathrm{~s}^{-1}$. This will result in a significantly higher levels of background radiation. The Cathode Strip Chambers (CSCs) are planned to operate throughout the HL-LHC era. Data from CSCs are used to provide online trigger primitives for the CMS Muon trigger, as well as hits and track segments used for CMS High Level Trigger (HLT) and for offline reconstruction of muon segments. The study presented here addresses the stability of CSC L1 trigger primitives based on the response of two CSCs that are undergoing high radiation exposure at the CERN Gamma Irradiation Facility (GIF++) [2].

\subsection{The CMS Muon System}

The Muon system is a key component of the CMS detector [3] with three primary objectivesmuon triggering, identification and momentum measurement. It consists of ionization gas detectorsDrift Tubes (DTs), Resistive Plate Chambers (RPCs) and CSCs. The DTs cover the barrel region whereas the CSCs cover the endcap region, and RPCs cover both.

\subsection{Cathode Strip Chambers (CSCs)}

The CSCs are trapezoidal Multi Wire Proportional Chambers (MWPC) [4] with six layers. The strips are oriented along the radial direction to measure the $\phi$ coordinate whereas the wires, which measure the $r$ coordinate, are orthogonal to the strips [5] (Figure 1). The gas gaps are filled with a gas mixture of $\operatorname{Ar}(40 \%), \mathrm{CO}_{2}(50 \%)$ and $\mathrm{CF}_{4}(10 \%)$. A muon passing through the gas gap causes ionization of gas molecules which results in an avalanche of electrons around the wires and an induced charge on the cathode strips.

In CMS Muon endcaps, the CSCs are arranged in a series of concentric rings centered on the beam line. ME1/1 ${ }^{1}$ chambers, of all the CSCs, cover the most forward region $(1.6 \leq|\eta| \leq 2.4)^{2}$ and hence, they are exposed to the highest rate from background radiation. ME2/1 chambers are the next-closest to the beam line after ME1/1.

The digital CSC electronics of anode and cathode readouts searches for muon candidates every bunch crossing ( $25 \mathrm{~ns}$ ). The Anode Local Charged Track (ALCT) board finds wire hits that are aligned across at least four out of six layers. Similarly, the Cathode Local Charged Track (CLCT) board finds strips hits that are aligned across at least four out of six layers. A comparator circuit determines the position of the hits with half-strip accuracy. The information from ALCT and CLCT is combined to define a 2-dimensional Local Charged Track (LCT). Upon arrival of a Level-1 (L1) trigger, matching the ALCT/CLCT generated by the chamber, data in the corresponding sampling time window are digitized and readout.

\section{Experimental Procedure}

\subsection{Experimental Setup}

The CERN GIF++ facility is equipped with a $14 \mathrm{TBq}{ }^{137} \mathrm{Cs}$ source that emits $662 \mathrm{keV}$ photons. A muon beam is provided by the CERN SPS accelerator. A large scintillator $(40 \mathrm{~cm} \times 30 \mathrm{~cm})$ is placed outside the bunker at each end. At GIF++, the two CSCs (ME1/1 and ME2/1) are placed inside a bunker at a distance of $2.4 \mathrm{~m}$ and $3.2 \mathrm{~m}$ from the irradiation source, along a muon beam line (Figure 2a). A third scintillator $(10 \mathrm{~cm} \times 10 \mathrm{~cm})$ is placed near ME2/1.

\footnotetext{
${ }^{1}$ The CSCs are labelled according to their position within the CMS Muon Endcap, i.e. ME2/1 refers to the CSCs in the first ring in the second disk.

${ }^{2} \eta=-\ln (\tan (\theta / 2))$, where $\theta$ is the polar angle.
} 

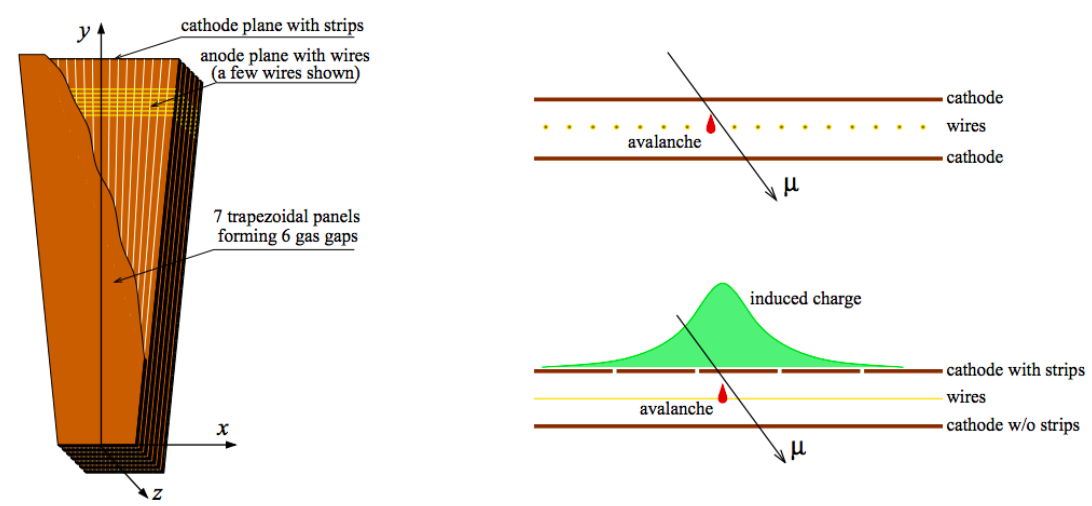

Figure 1: A schematic of CSC (left) with a cutout of the top layer. Working principle of CSCs (right). A muon crossing the gas gap ionizes gas molecules, and the electrons drift towards the anode wire, where the avalanche is caused by the high electric field. This induces charge on the cathode strips and the location of the particle can be precidely measured from the distribution of charge induced on the strips.

The operational High Voltages ${ }^{3}$ of both CSCs are adjusted to provide an equalized gas gain, i.e. same gas gain across all layers. We define the "Trim Mean" charge as the mean of the truncated distribution ${ }^{4}[6]$ of the cathode charges measured in a single layer of a CSC, which we use as a proxy for gas gain. The working point HV settings for the CSCs at the CMS Muon endcap corresponds to a "Trim Mean" charge of 300 ADC counts.

The irradiation source provides a high radiation environment leading to increased background hits in the chambers. The radiation intensity can be varied by using a combination of attenuating filters. The higher the source intensity the higher the current measured in the wire planes of the CSCs at GIF++. The wire current per layer in a ME1/1 chamber installed in CMS, measured during Run 2 (2018), is shown in Figure $2 b$ as a function of LHC instantaneous luminosity. The LHC instantaneous luminosity equivalent to the irradiation source intensity can be inferred from the linear relation (Figure 2b).

\subsection{Analysis Strategy}

A coincidence of signals from the three scintillators produces a trigger signal corresponding to a muon passing through the CSCs. Upon arrival of the trigger, the data from both chambers are read-out. One of the two chambers is used as reference in the analysis. In order to select events containing single muons that passes through both chambers, and are not accompanied by any showers, we use the following selection criteria:

1. An event must have exactly 1 ALCT, 1 CLCT and 1 correlated LCT within the fiducial volume of the reference chamber. This requirement is imposed to select events with a single muon.

2. An event can have at most 20 hits in time with the muon, in comparators and wires within the fiducial volume of the reference chamber. The requirement rejects events with single muons accompanied by showers $(\approx 10 \%$ of all triggers $)$.

\footnotetext{
${ }^{3}$ See [5] for detail description of HV distribution in CSCs.

${ }^{4}$ The distribution is truncated when the the area under the curve becomes $70 \%$ of the total area.
} 
The efficiency for a given trigger primitive TP (ALCT, CLCT, LCT) can be defined as

$$
\varepsilon_{\mathrm{TP}}=\frac{\mathbf{n}}{\mathbf{N}},
$$

where $\mathbf{N}$ is the number of single muon events in the refrence chamber and $\mathbf{n}$ is the number of events in which there is at least $1 \mathrm{TP}$ in the fiducial volume of the test chamber.

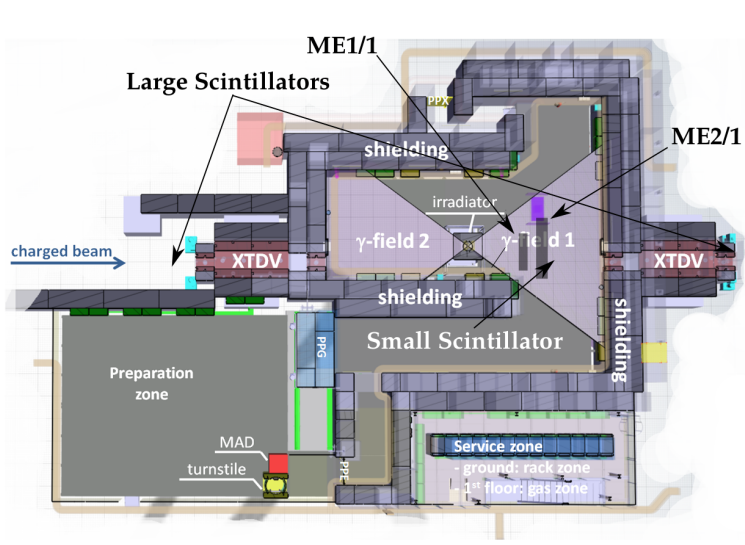

(a)

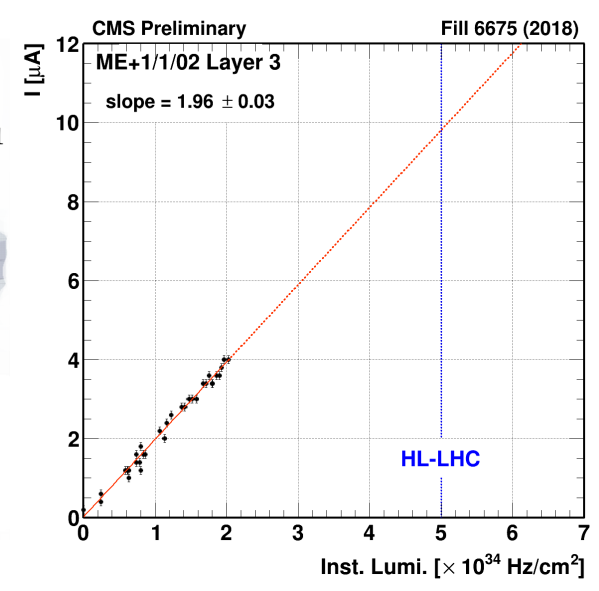

(b)

Figure 2: (a) A schematic layout of experimental setup GIF++. (b) ME1/1 current as a function of the LHC instantaneous luminosity. The line (red) represents a linear fit.

\section{Results}

Figure 3 shows ME1/1 Trigger Primitive efficiencies. The efficiency increases with increasing gas gain and reaches a plateau (Figure $3 \mathrm{a}$ and Figure 3b). An increase in the source intensity causes a decrease in the efficiency (Figure $3 \mathrm{c}$ and Figure $3 \mathrm{~d}$ ) due to increasing background hit rate. For the HL-LHC luminosity of $5 \times 10^{34} \mathrm{~cm}^{-2} \mathrm{~s}^{-1}$, the average current per layer from the linear fit was found to be $10 \mu \mathrm{A}$ in ME1/1 and $15 \mu \mathrm{A}$ in ME2/1.

\section{Conclusion}

The Trigger Primitive efficiencies of the CSCs at HL-LHC like environment are reasonably high. The marginal losses are caused by the increased background hits in the chambers. The loss of LCT efficiency with increasing background radiation can be attributed to the a slight inefficiency in CLCT comparator logic in both chambers. In ME2/1, additional LCT losses are caused by inefficiency of the LCT finding logic in matching an ALCT to the corresponding CLCT. The estimated LCT efficiency at HL-LHC-like radiation environment is $99.4 \%$ for ME1/1 and $98.2 \%$ for ME2/1. 


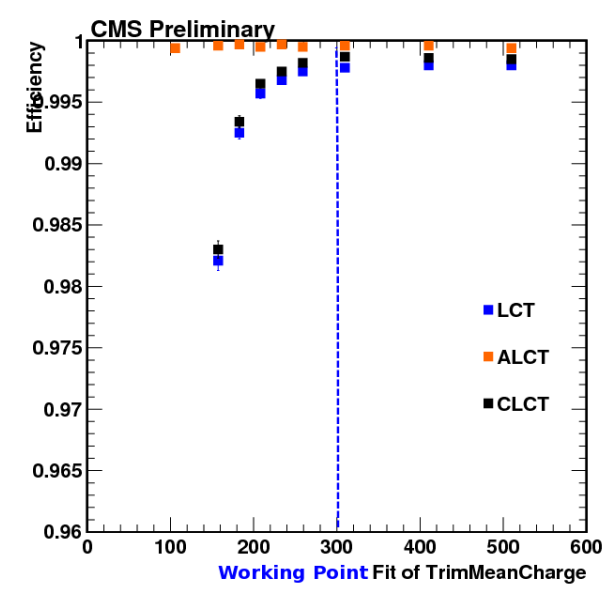

(a)

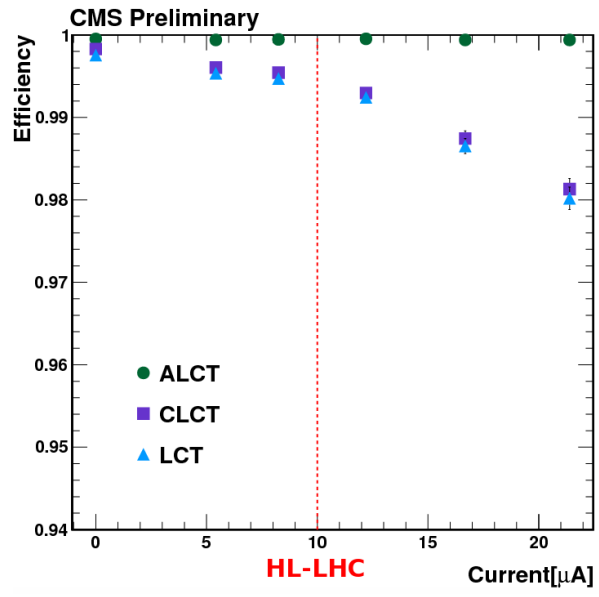

(c)

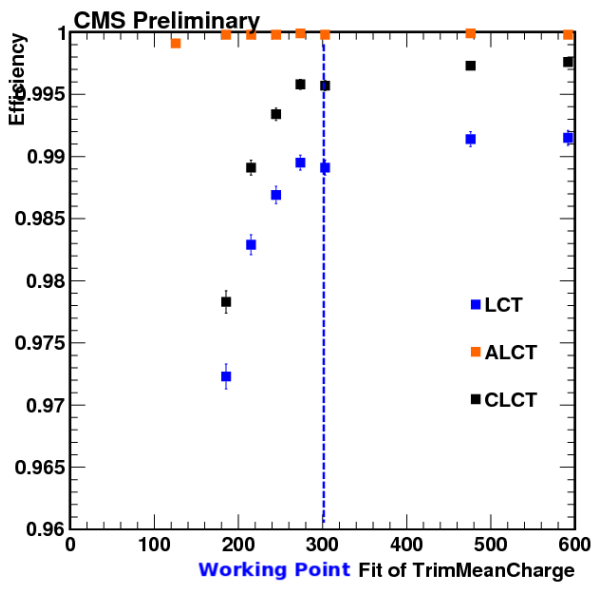

(b)

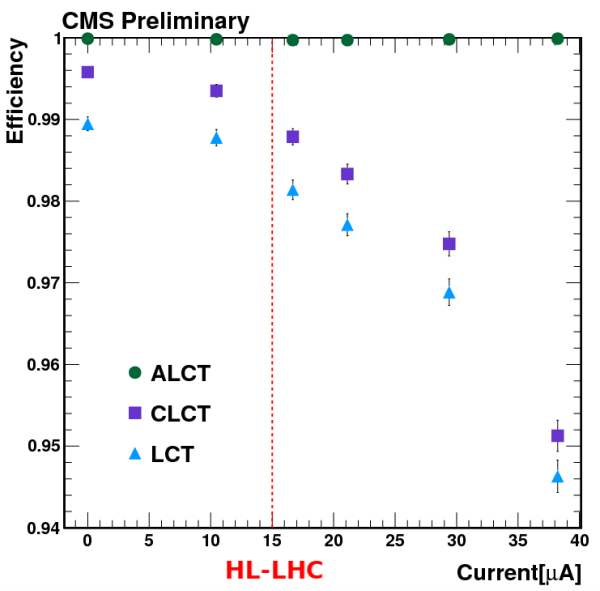

(d)

Figure 3: (a) ME1/1 efficiency as a function of gas gain. The horizontal axis represents the average "Trim Mean" charge. The nominal working point is indicated by the vertical line (blue). (b) ME2/1 efficiency as a function of gas gain. (c) ME1/1 efficiency as a function of wire current per layer. The vertical line (red) indicates the wire currents corresponding to HL-LHC luminosity. (d) ME2/1 efficiency as a function of current per layer.

\section{References}

[1] High-Luminosity Large Hadron Collider (HL-LHC) Technical Design Report V. 0.1, DOI: http://dx.doi.org/10.23731/CYRM-2017-004.

[2] M.R. Jaekel, et. al., CERN GIF++ : A new irradiation facility to test large-area particle detectors for the high-luminosity LHC program, 2014, PoS(TIPP2014)102.

[3] CMS Collaboration, The CMS experiment at the CERN LHC, JINST 3:S08004, 2008

[4] G. Charpak, et. al., Nuclear Instrumentation and Methods 62 (1968) 202.

[5] CMS Collaboration, The Muon Project Technical Design Report, CERN/LHCC 1997-032, 1997.

[6] Landau L., On the energy loss of fast particles by ionization, J. Phys. (USSR). 8: 201, 1994. 